\title{
Fatal case of disseminated infection with the turtle bacillus Mycobacterium chelonae
}

\author{
J Paul, C Baigrie, D V Parums
}

\begin{abstract}
An apparently immunocompetent 78 year old woman presented with confusion, subcutaneous abscesses, and lesions of the nasopharynx. Gram positive, acid fast bacilli were isolated from her blood after 10 days' incubation. She was treated with trimethoprim-sulphamethoxazole for presumed disseminated nocardiasis but deteriorated and died. A post mortem examination showed skin and pulmonary lesions and endomyocardial fibrous plaques. Organisms isolated from the skin and lung were indistinguishable from those cultured from the blood. The organism was subsequently identified as Mycobacterium chelonae. Primary pulmonary infection and disseminated disease are rarely caused by this organism and bacteraemia is seldom documented.

The clinical presentation and bacteriological and histological findings are difficult to differentiate from those of disseminated nocardiasis. Isolation of the organism may fail without prolonged incubation of initial cultures and there is a danger of its being dismissed as medically unimportant. Diagnosis is further hampered because large pulmonary foci may be poorly revealed by conventional radiological examination of the chest.
\end{abstract}

Mycobacterium chelonae is one of the so-called "rapidly-growing mycobacteria" and closely resembles $M$ fortuitum, the other medically important member of the group. Once isolated, these bacteria will grow within one or two days on most routine media-unlike most other clinically important mycobacteria-but initial isolation may take from 10 to 30 days. ${ }^{1} M$ chelonae is widespread in soil and water ${ }^{2}$ and causes disease in amphibians, ${ }^{3}$ reptiles ${ }^{4}$ and occasionally in man. The spectrum of human disease varies from local abscess formation (often related to direct inoculation) to focal pulmonary disease, postsurgical sepsis and potentially lethal disseminated illness. Death is a rarely reported outcome of this infection.

Diagnosis is much hampered in the laboratory by the initial slow growth of the organism and its close resemblance to Nocardia spp (being aerobic, Gram positive, acid fast, and variably coryneform or filamentous in appearance). Clinically, diagnosis is also hampered by the considerable overlap between the spectra of disease generated by rapidly growing mycobacteria and Nocardia spp.

\section{Case report}

A 78 year old woman presented with anorexia, weakness, and shortness of breath. She had a history of congestive cardiac failure and chronic obstructive airways disease. On admission she was confused, cyanosed, and peripherally oedematous. There were large fluctuant subcutaneous swellings on the limbs and on the face, causing distortion of the nose. Some small necrotic, apparently embolic lesions were present in the pulps of the fingers. Results of routine investigations included haemoglobin $14.3 \mathrm{~g} / \mathrm{dl}$, white cell count $14 \times$ $10^{9} / 1$, with $93 \%$ polymorphs, and an erythrocyte sedimentation rate $/ 8 \mathrm{~mm} /$ first hour. Blood cultures were taken. Chest radiographs showed patchy shadowing of both lower zones. She was treated initially with intravenous cefuroxime followed by oral amoxycillin for presumed chest infection.

After 10 days of incubation of blood cultures (Bactec) Gram positive rods resembling Propionibacterium sp were seen. When subcultured on to blood agar and incubated aerobically at $37^{\circ} \mathrm{C}$, convex, opaque, grey colonies $1 \mathrm{~mm}$ in diameter became apparent within 24 hours. Gram staining showed the organism to have attained a filamentous appearance. Modified Ziehl-Neelsen staining showed acid fastness. Similar organisms were seen and cultured from a cutaneous lesion aspirate.

A presumptive diagnosis of disseminated nocardiasis was made and treatment with intravenous trimethoprim-sulphamethoxazole was started. However, the patient died within 24 hours of treatment having been started. An isolate of the organism was subsequently identified by the Mycobacterial Reference Laboratory, Dulwich, London, as $M$ chelonae. In vitro sensitivity testing showed it to be resistant to all commonly used antibiotics including those recommended for treatment of this infection such as amikacin, doxycycline, trimethoprim, sulphonamides and imipenem. 
Figure 1 Macroscopic photograph taken of the right lung, sliced longitudinally down the middle. Note the large, confluent, necrotic abscesses.

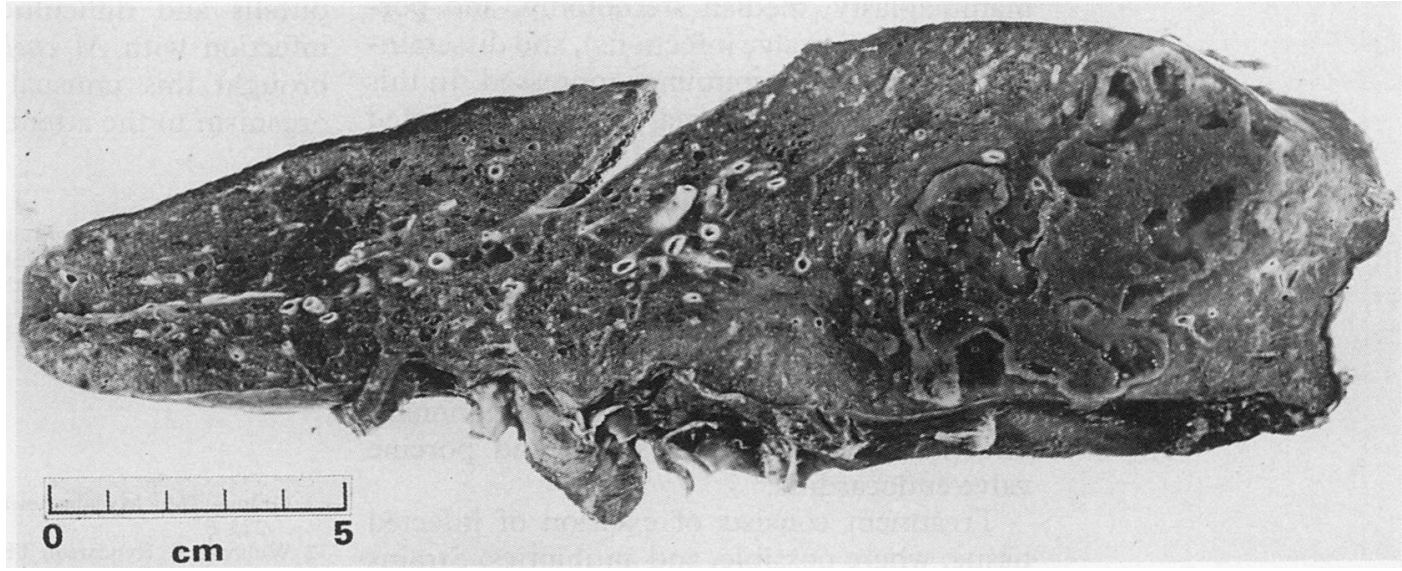

\section{Pathological findings}

The body was that of a thin, elderly woman with extremely poor dentition and pitting oedema up to the knees. The nose was misshapen and both nostrils were occluded by haemorrhagic, crusting skin lesions. Similar lesions were noted on the bridge of the nose, left cheek, around the right knee and on the pulps of the fingers. Two well circumscribed pulp infarcts were also present on the right thumb.

Bilateral pleural effusions were present. The right lower lobe was firmly adherent to the chest wall. The left lung weighed $680 \mathrm{~g}$, the right lung weighed $515 \mathrm{~g}$. On slicing the right lung, there were numerous confluent abscess cavities in the lower and middle lobes which were surrounded by areas of hyperaemia (fig 1).

The heart was enlarged $(515 \mathrm{~g})$ with left ventricular hypertrophy $(17 \mathrm{~mm}$ wall thickness) and dilatation. The cardiac valves were normal. Several white, fibrous endomyocardium plaques were presnt in the left and right ventricles. The remaining organs, including the brain, were normal.

Histological examination of the skin and lungs showed confluent, necrotic, haemor-

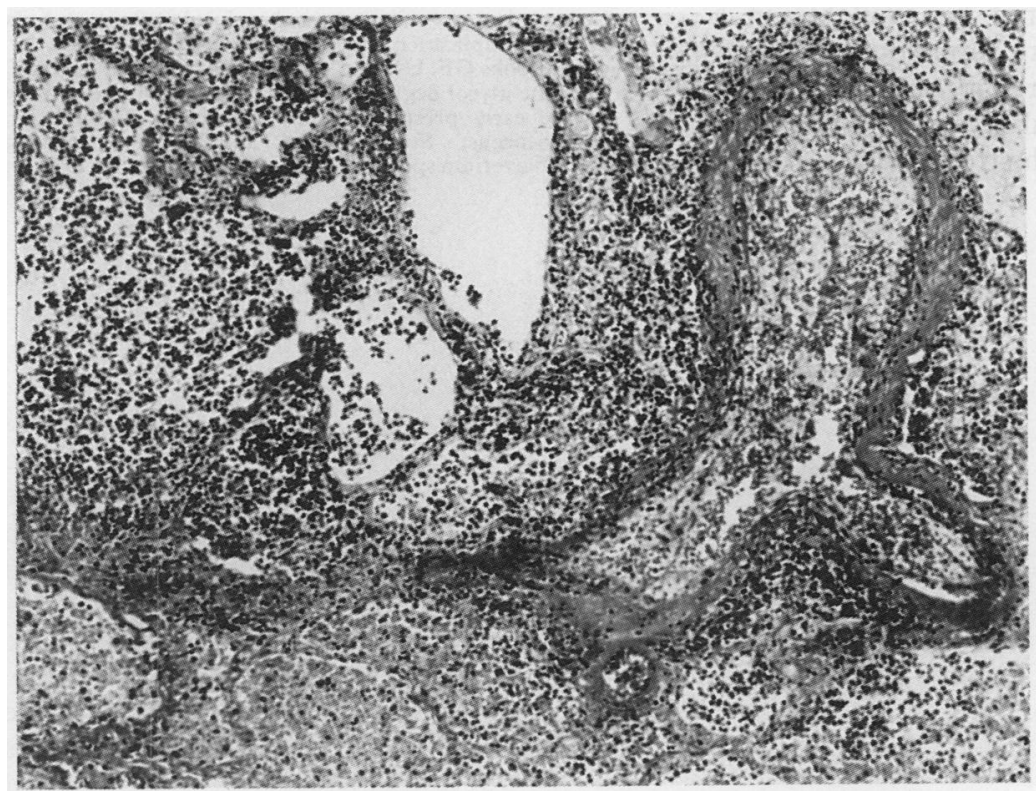

Figure 2 Histological appearances of the lung with necrosis centred around vessels, showing little evidence of granulation tissue or fibrosis (Haematoxylin and eosin). rhagic lesions, often involving vessels and showing remarkably little evidence of healing or fibrosis (fig 2). Granulation tissue was seen around intramyocardial vessels and numerous endocardial fibrotic plaques were present.

Despite the absence of brain disease, both the necropsy and subsequent histological findings were considered to be consistent with a diagnosis of disseminated nocardiasis.

\section{Discussion}

$M$ chelonae was isolated from sea turtles, Chelona corticata, by Friedman, ${ }^{4}$ who called it the "Schildkröten tuberkelbazillus". He advocated the use of this organism for prophylactic vaccination against tuberculosis, but this was commonly found to cause abscesses at the injection site. ${ }^{1}$ The organism has been referred to previously using various names, including the invalidly published $M$ fried$m{ }^{2} n i^{1}$ and the grammatically incorrect $M$ chelonei. ${ }^{5}$

The rapidly growing mycobacteria $M$ chelonae and $M$ fortuitum are recognised causes of a wide variety of human infections including soft tissue and injection site abscesses, ${ }^{6-8}$ primary pulmonary disease, ${ }^{69}$ disseminated disease, ${ }^{610}$ sternotomy infection and prosthetic valve endocarditis. ${ }^{611} 12$ They are widespread environmental organisms, which can be cultured from soil and water, ${ }^{2}$ but which seldom infect man. Localised tissue infection and abscess formation can occur following direct inoculation, as may take place during gardening injuries or injection of contaminated solutions. ${ }^{10}$ The organisms can survive in antibiotic media and can be isolated from bioprosthetic heart valves stored in such solution ${ }^{13}$ and can cause prosthetic valve endocarditis. ${ }^{11}$ Primary pulmonary disease is a rare but well described phenomenon. ${ }^{914}$ Disseminated disease may occur in debilitated or immunocompromised patients, or in chronic, untreated disease in the immunocompetent.

Wallace ${ }^{6}$ reviewed 125 cases of rapidly growing mycobacterium infection, consisting mostly of immunocompetent individuals with localised infection, penetrating injury site abscesses, and more rarely, cervical lymphadenitis and keratitis. Other categories of disease included postsurgical sepsis (augmentation 
mammaplasty, median sternotomy, and porcine prosthetic valve infections), and disseminated disease in the immunosuppressed. In this review, ${ }^{6}$ primary pulmonary disease accounted for 24 of 125 cases (17 of $24 \mathrm{M}$ chelonae), $63 \%$ of the patients being female with a mean age of 58 years. Only $38 \%$ had pre-existing illness. Bacteraemia was recorded in only 12 of 125 cases and was associated with prosthetic valve infection, disseminated disease, line sepsis or chronic chemotherapy. Death is a rarely reported outcome and is associated with pulmonary infection, sternal wound sepsis and porcine valve endocarditis. ${ }^{6}$

Treatment consists of excision of infected tissue, where possible, and antibiotics. Strains commonly exhibit in vitro resistance to most antibiotics, but those that have been successfully used include amikacin, doxycycline, and sulphonamides. ${ }^{15}$ Infection is rarely fatal, but endocarditis, sternal wound infection, and pulmonary and disseminated disease carry poor prognoses. When detected early enough, localised pulmonary infection may be treated adequately by excision of the affected lobe. ${ }^{9}$

There are many diagnostic problems associated with $M$ chelonae infection, among them a general lack of awareness which may thwart diagnosis. Large pulmonary lesions may not be readily discernible radiologically. ${ }^{6}$ The organism is apt to cause slowly progressing chronic disease lacking distinctive features. Infective endocarditis of a bioprosthetic heart valve has become clinically evident as late as three years after implantation. ${ }^{11}$ The slow primary isolation time of the organism may result in its being missed using routine culture methods. When cultured, the organism may be mistaken for a medically unimportant diphtheroid or discounted for not being $M$ tuberculosis. Because $M$ chelonae is widespread in water, isolates may be deemed falsely positive. ${ }^{16}$

As in this case the clinical presentation and cultures may be confused with those of nocardiasis. $M$ chelonae produces arylsulphatase, a usefully distinguishing feature from Nocardia spp, but testing may not be available in a routine laboratory. More sophisticated methods of identification are appropriate in the reference or research laboratory. ${ }^{17}$ We hope that this report has illustrated some of the pitfalls and difficulties in the diagnosis of infection with $M$ chelonae and that we have brought this unusual and, thus far, elusive organism to the attention of clinical pathologists.

We thank the staff of the Mycobacteria Reference Laboratory, Public Health Laboratory, Dulwich, London, for the identification and sensitivity testing of the isolates of $M$ chelonae. We also thank Professor J Grimley Evans of the Radcliffe Infirmary, thank Professor $J$ Grimley Evans of the Radcliffe In
Oxford, for permission to publish details of his patient.

1 Grange JM. Mycobacterium chelonei. Tubercle 1981;62: 273-6.

2 Wolinsky E, Rynearson TK. Mycobacteria in soil and their relation to disease-associated strains. Am Rev Respir Dis 1968;97:1032-7.

3 Mok WY, Carvalho CM. Occurrence and experimental infection of toads (Bufo marinus and B granulosus) with Mycobacterium chelonei subsp. abscessus. J Med Microbiol 1984;18:327-33.

4 Friedmann FF. Der Schildkröten tuberkelbazillus, seine Zuchtung, Biologie und Pathogenität. Zentralblatt für Bakteriologie, Parasitenkunde, Infectionskrankheiten und Hygiene, I. Orig 1903;34:647-62; 793-832.

5 von Graevenitz A, Berger U. A plea for linguistic accuracy. Int J Syst Bacteriol 1980;87:189.

6 Wallace RJ Jr, Swenson JM, Silcox VA, et al. Spectrum of disease due to rapidly growing mycobacteria. Rev Infect Dis 1983;5:657-79.

7 Jackson PG, Keen H, Noble CJ, Simmons NA. Injection abscesses due to Mycobacterium chelonei occurring in a diabetic patient. Tubercle 1981;62:277-9.

8 Hanson PJV, Thomas JM, Collins JV. Mycobacterium chelonei and abscess formation in tissues. Tubercle 1987; 68:297-9.

9 Tsukamura M, Nakamura E, Kurita I, Nakamura T. Isolation of Mycobacterium chelonei subspecies chelonei (mycobacterium borstelense) from pulmonary lesions of 9 patients. Am Rev Respir Dis 1973;108:683-5.

10 Azadian BS, Beck A, Curtis JR, et al. Disseminated infection with Mycobacterium chelonei in a haemodialysis patient. Tubercle 1981;62:281-4.

11 Rumisek JD, Albus RA, Clarke JS. Late Mycobacterium chelonei bioprosthetic valve endocarditis: activation of
implanted contaminant? Ann Thorac Surg 1985;39:277-9.

12 Wallace RJ Jr, Musser JM, Hull SI, et al. Diversity of sources of rapidly growing mycobacteria associated with infections following cardiac surgery. $J$ Infect Dis 1989; 159:708-16.

13 Laskowsi LF, Marr JJ, Spergona JF. Fastidious mycobacteria grown from porcine prosthetic-heart-valve cultures. N Engl J Med 1977;297:101-2.

14 Trulock EP, Bolman RM, Genton R. Pulmonary disease caused by Mycobacterium chelonae in a heart-lung transplant recipient with obliterative bronchiolitis. Am Rev Respir Dis 1989;140:802-5.

15 Sanders WE Jr, Howowitz EA. Other mycobacterial species. In: Mandell GL, Douglas RG, Bennett JE, eds. Principles and practice of infectious diseases. 3rd edn. 1990:331-98.

16 Nye K, Chadha DK, Hodgkin P, et al. Mycobacterium chelonei isolation from broncho-alveolar fluid and its chelonei isolation from broncho-alveolar fluid and
practical implications. J Hosp Infect 1990;16:257-61.

17 Fiss E, Brooks GF. Use of a siderophore detection medium, ethylene glycol degradation, and $\beta$-galactosidase activity in the early presumptive differentiation of Nocardia, Rhodococcus, Streptomyces, and rapidly growing Rhodococcus, Streptomyces, and rapidly growing
Mycobacterium species. J Clin Microbiol 1991;29:1533-5. 